\title{
Variação de Soluções na Geometria com a utilização do GeoGebra
}

\author{
Variation of Geometry Solutions with the use of GeoGebra
}

MARCOS PAULO MESQUITA DA CRUZ ${ }^{1}$

IVAN DE OLIVEIRA HOLANDA FILHO ${ }^{2}$

http://dx.doi.org/10.23925/2237-9657.2019.v8i2p078-101

\begin{abstract}
RESUMO
O presente artigo tratou de uma solução alternativa oficial para a questão da Obmep do ano de 2017 primeira fase, nível três com assistência do software GeoGebra na construção das figuras. A questão aborda o assunto de Geometria Plana que disponibilizou uma variação de assuntos $e$ conceitos matemáticos para sua resolução: Semelhança de Triângulos, Mediana, Bissetriz, Teorema de Pitágoras, Trigonometria, etc., estudados no ensino Fundamental e Médio. Assim, objetivou-se uma solução com mais qualidade em detalhes e nos tópicos ligados aos assuntos de ensino, matemática e tecnologia educacional. Constatou-se que o GeoGebra é uma ferramenta que facilita o aprendizado da matemática e que os métodos de ensino precisam de avaliação na tentativa de encontrar a melhor escolha em sala de aula. Por fim, o artigo apresentou-se, de maneira elegante, que a criatividade é fundamental para o ser humano, pois acarretou treze soluções distintas e com a pesquisa realizada proporcionaram um livro.
\end{abstract}

Palavras Chaves: Ensino de Matemática, Geometria, GeoGebra, Tecnologia e Soluções.

\section{ABSTRACT}

The present article dealt with an alternative solution to the official one for the 2017 first phase, three standard issue with the assistance of GeoGebra software in the construction of the figures. The question deals with the subject of Plane Geometry, a perspective of some questions and mathematical concepts for its resolution: the triangle similarity, the median, the bisector, the Pythagorean theorem, trigonometry, etc., those studied in School. Thus, a solution with more quality in details and the problems of teaching, mathematics and educational technology were objectified. It was found that GeoGebra is a tool that facilitates the learning of mathematics and that teaching methods are under evaluation to find a better choice in the classroom. Finally, the article presented, in an elegant way, that creativity is fundamental for the human being, because it resulted in thirteen different solutions and with the research performed provided a book.

Keywords: Teaching Mathematics, Geometry, GeoGebra, Technology and Solutions.

\footnotetext{
${ }^{1}$ Mestre em Economia Rural (UFC), Bacharel em Ciências Contábeis (UECE) e em Engenharia Metalúrgica (UFC). Professor de cursos técnicos e escolas particulares de ensino. e-mail: marcos_paulo_mesquita@hotmail.com

${ }^{2}$ Pós-Graduação em Ensino de Matemática (UNIATENEU), Licenciado em Matemática (UECE). Professor da Rede Básica de ensino em Maracanaú e do estado do Ceará. e-mail: ivanfilho@ymail.com https://orcid.org/0000-0002-6368-9971
} 


\section{INTRODUÇÃO}

$\mathrm{Na}$ atualidade, o ensino nas escolas públicas e particulares sofre por profundas transformações em função da adequação das melhores propostas de aprendizagem. A tarefa passa a ser um desafio quando a realidade das escolas detém uma grande heterogeneidade pelas condições de trabalho, estruturas físicas, tecnológicas e etc., e a preocupação na preferência de método mais interessantes para lecionar.

No dia 05.12.2018 foi apresentada uma nova Base Nacional Comum Curricular (BNCC) que está em fase de homologação e que prevê apenas matemática e português como sendo obrigatórias nos 3 anos. Nesta mesma data foi anunciado que as escolas poderiam escolher $40 \%$ da carga horária do ensino médio e os outros $60 \%$ serão estabelecidos pela BNCC. Observa-se que todas essas mudanças ocorridas no ensino médio são por diversas razões. Uma razão importante é o desprezo do jovem por esse modelo atual, dado que o conjunto de 13 disciplinas letivas induzem ao conhecimento superficial das matérias e o desagrado por assistir aulas de temas que o jovem possui pouca identidade. Esse cenário é consequência para a desistência de 700 mil alunos por ano no ensino médio. Economicamente, o país perde 3,7 bilhões por ano com a evasão escolar, segundo Mozart Ramos Neves, diretor do Instituto Ayrton Senna, ex-secretário de Educação de Pernambuco e ex-reitor da Universidade federal de Pernambuco (HOLANDA FILHO; CRUZ, 2019, p. 18).

Nesse quadro, apresentam-se gradualmente o uso de softwares e aplicativos como uma possibilidade eficiente para propagar o ensino de matemática, por exemplo, em assuntos de geometria que facilitam a aprendizagem de álgebra, a interpretação de problemas e figuras. Para Fainguelernt e Nunes (2012, p. 116) percebe-se a relação da geometria com a aprendizagem:

\footnotetext{
A importância de se investigar a introdução da geometria desde o primeiro ano do ensino fundamental até o ensino médio como exploração do espaço e como uma estrutura lógica, é justificada pelo papel formativo que essa disciplina desempenha na
} 


\begin{abstract}
construção do conhecimento nas diferentes áreas e no desenvolvimento da habilidade espacial dos aprendizes. Essa importância da geometria na construção do conhecimento matemático é confirmada pelos resultados de pesquisas realizadas desde a década de 1970. Pode-se afirmar que a geometria oferece um vasto amplo de ideias e métodos de muito valor, quando se trata do desenvolvimento intelectual do aluno do seu raciocínio e da passagem da instituição de dados concretos e experimentais para os processos de abstração e generalização.
\end{abstract}

Compreende-se que a geometria é a ciência que estuda o espaço, pois trabalha com formas e medições, sendo uma ferramenta importante para diversas áreas e de grande significativa para os alunos desenvolverem suas aptidões, conceitos e o seu raciocínio geométrico (ROGENSKI; PEDROSO, 2007).

Para Dziadzio e Vankan (2016), as experiências da prática pedagógica mostram comuns as dificuldades de alunos em solucionarem problemas quando não possuem a base de geometria. Isso é comprovado em assuntos de geometria espacial, onde o cálculo de áreas e volumes não são tão bem compreendidos entre os alunos. Muitas vezes, os descompassos do ensino são vindos da difículdade não suprida do ensino fundamental em não se trabalhar estruturalmente o ensino de forma conexa com a didática pedagógica da matemática com as demais áreas de conhecimento.

Neste contexto, o presente estudo se propõe na solução de uma questão da Olimpíada Brasileira de Matemática das Escolas Públicas (Obmep) por meio de duas possibilidades distintas, despertando a curiosidade e questionamento de quantas formas distintas seria possível resolver a questão. Quais os assuntos que poderiam resolvê-la? O número de soluções seria alto por meio da Geometria?

$\mathrm{Na}$ organização do trabalho, o mesmo está dividido em mais quatro seções, além da introdução. Na seção revisão de literatura faz-se a abordagem dos principais tópicos para o desenvolvimento do estudo, com perspectiva para a ciência matemática e o uso da tecnologia educacional para resolução de questões 
tais como da olimpíada brasileira de matemática das escolas públicas. Logo em seguida, na seção 3, apresenta-se a questão que iniciou todo o desenvolvimento do trabalho com as duas soluções e, por fim, apresentam-se as considerações finais e as referências.

\section{FUNDAMENTAÇÃO TEÓRICA}

Neste capítulo será apresentada uma síntese dos principais tópicos para o desenvolvimento do estudo, com perspectiva para a ciência matemática e o uso da tecnologia educacional para resolução de questões tais como da olimpíada brasileira de matemática das escolas públicas.

\subsection{Mecanismos para o Ensino das Ciências}

O desafio de elaborar mecanismos, métodos e táticas para um ensino focado no aprendizado é a característica que se mantém comum na oferta de disciplinas disponibilizadas nas escolas do ensino médio. A preocupação em lecionar as ciências tais como Física, Química, Biologia e Matemática exigem dos docentes maior entendimento e compreensão do universo educar.

Previamente, precisa-se definir o termo "Ciência" e seu vínculo ao ensino. Pelo dicionário Aurélio de Ferreira, temos que ciência significa:

\footnotetext{
1- Conhecimento ou saber excessivo conseguido pela prática, raciocínio ou reflexão. 2-Reunião dos saberes organizados obtidos por observação, pesquisa ou pela demonstração de certos acontecimentos, fatos, fenômenos, sendo sistematizados por métodos ou de maneira racional: as normas da ciência. 3Análise, matéria ou atividade que se baseia numa área do conhecimento: ex. a ciência da matemática (Dicionário Aurélio).
}

Observa-se que o saber possui diversas fontes de se obtê-lo e que certos conteúdos constatados da forma empírica são direcionados de maneira sistêmica e com pouco engajamento nos processos de aprendizagem. Para Saraiva et al. (2018, p. 39) a visão de ciência fica mais ampla quando se profere a importância para a sociedade. 
As Ciências não são atividades neutras e o seu desenvolvimento está diretamente imbricado com transformações sociais, políticas, econômicas, manifestações culturais e ambientais. Portanto, o estudo de ciências não se reporta somente aos intelectuais, mas ao cidadão comum que procura evoluir junto com a sociedade.

Em Vasconcelos e Araújo (2018, p. 53) apresenta-se a contextualização do ensino na década de 80 e a preocupação na formação do cidadão.

O reconhecimento de que o processo de ensino de ciências apresenta fragilidades, levou há mais de três décadas, à elaboração de metodologias de ensino mais progressistas, que indicassem a viabilidade de se conseguir a produção do conhecimento e a formação de um cidadão crítico, com capacidade de analisar, compreender e fazer uso desse conhecimento em seu dia a dia.

Continuando em Saraiva et al. (2018, p. 39), tem-se a discriminação entre as épocas e a atuação das novas teorias.

\begin{abstract}
Acredita-se que a concepção passada, relacionada com a percepção do presente conjecturam a aparição de uma complementação entre uma época e outra. Logo, a concepção, abordada ao Ensino das Ciências, leva a construir conhecimentos em que teorias atuais sejam resultado da colaboração de ideias científicas do passado ocorridas em períodos distintos.
\end{abstract}

Neste contexto, verifica-se a sugestão de soluções por métodos de casos práticos:

Em Lima et al. (2018) descreve as contribuições da atividade prática laboratorial para a aprendizagem significativa e como despertou o interesse dos aprendizes, facilitando a assimilação de conceitos da disciplina de química.

No trabalho de Rodrigues e Alves (2018) a metodologia de aprendizagem adotada é a análise e relevância da criação/produção, montagem e distribuição de fanzines para o campo educativo escolar, com foco no ensino de biologia. A hipótese é de que as práticas zínicas em espaços escolares podem ampliar as possibilidades de aprendizagem dos conteúdos biológicos e (re)aproximar ciências e artes. 
Já em Silva et al. (2018) a proposta de ensino foi uma atividade prática realizada por alunos do curso de mecânica industrial, utilizando uma plataforma robótica com o objetivo de facilitar a compreensão de conceitos básicos de cinemática, que são considerados os mais importantes quando o aluno faz a transição do ensino fundamental para o ensino médio.

Por fim, no ensino de matemática, a aplicabilidade de tecnologias na educação e a variedade de recursos didáticos como vídeos e softwares ascende-se paulatinamente. Lima e Pereira (2018) realizaram o experimento em avaliar as percepções dos professores em formação sobre o uso de vídeos como recurso didático para o ensino da matemática em escolas públicas, enquanto, Lima et al. (2018) e Santos, Alves e Vasconcelos (2018) adotam a metodologia Engenharia Didática proporcionada pelos recursos tecnológicos do software GeoGebra em aulas de cálculo no ensino superior.

A partir da hipótese sugestiva para o desenvolvimento deste artigo, será abordado a narrativa da disciplina de matemática pelo tópico de Geometria, que é um conhecimento amplamente introduzido desde as séries iniciais até o ensino médio.

\subsection{Matemática e sua Relevância}

O ensino da matemática possui um valor imensurável para a vida de uma pessoa. O desenvolvimento do raciocínio dedutivo, a estruturação do pensamento, o modo de processar as ideias para a solução de um problema e a presença nas diversas atividades humanas são alguns exemplos da maneira que o aprendizado desta disciplina é benéfico para um aluno e a sociedade no geral.

Em contraparte, muitos conteúdos de matemática não possuem planejamento pedagógico adequado para uma melhor didática, por isso, alguns temas não são explanados e compreendidos em sala de aula no ensino fundamental (PIRES, 1993).

Nesse cenário, as discussões de Lorenzato (1995, p. 5) e Duval (1988, p. 57) frisam que os problemas de Geometria apresentam uma grande originalidade em relação a muitas outras tarefas que podem ser propostas aos estudantes, possibilitando a contextualização e interdisciplinaridade para aplicação em outros 
tópicos matemáticos.

As dificuldades em ensinar, muitas vezes, passa pela relação do professor com o saber matemático e de sua experiência profissional. A primeira problemática que se encontra é que muitos professores do ensino fundamental não possuem os conhecimentos necessários na Geometria e não sabem compactuar em suas atividades pedagógicas. A segunda razão deve-se a falta de material didático eficiente deixando entendível que, pela falta de abordagem correta em sala de aula, o fracasso da geometria e o déficit no ensino penduram-se de geração à geração (PEREZ, 1991; PAVANELLO, 1993).

Somado a isso tudo há algumas décadas os livros valorizavam pouca a Geometria, pois muitos conteúdos eram vistos somente no final do livro, porém, as novas edições se preocupavam com o conteúdo e a organização didática. Entretanto, as dificuldades prevaleceram para aqueles que eram daquela realidade de ensino. Por isso, a importância em dar significado em alguns momentos do que é visto, ou seja, mostrar a aplicabilidade no ensino de Geometria para aumentar a aprendizagem tanto no Ensino Fundamental como no Médio.

A palavra Geometria vem do grego e significa medida da terra e a sua aplicação é vista em toda parte, sendo fundamental em diversos cursos de nível superior como Arquitetura, Engenharia, Matemática, Geologia e entre outros. Para Devlin (2008, p. 100) a Geometria demonstra-se relevante na vida dos docentes tanto pelo pensamento matemático adquirido como para a sociedade:

A exposição ao pensamento matemático formal é importante pelo menos por duas razões. Primeira um cidadão no mundo de hoje, baseado na matemática, deveria pelo menos ter uma ideia geral de uma das maiores contribuições à sociedade. Segunda, uma pesquisa levada a efeito pelo departamento de Educação dos Estados Unidos em 1997(o relatório Riley) mostrou que os estudantes que cursaram geometria no ensino médio saíram-se muito melhor nos exames para o curso superior, e também nos seus cursos universitários, do que aqueles que não estudaram a matéria, independentemente das matérias estudadas no curso superior. Como destacaram os organizadores da pesquisa, o principal fator não foi o grau de competência que os alunos de geometria mostraram neste curso.Apenas o fato de terem 
estudado a matéria já lhes dá uma enorme vantagem em todos os cursos.

A análise que exige destaque são as preocupações na educação para outros tópicos matemáticos. Nota se que o ensino de área das figuras planas, por exemplo, o conteúdo é repassado apenas por meio de fórmulas e não possui uma preocupação na metodologia, na representação gráfica e na contextualização dos exercícios trabalhados em sala de aula. Ainda prevalece o ensino por métodos mecânicos que se apresentam altamente desgastados e pouco compreendidos entre os alunos (SANTOS; JUCÁ, 2014).

Para o desenvolvimento do conceito de áreas e figuras planas, o raciocínio de Chiummo (1998, p. 31) evidencia que:

Se o conceito de área for repassado através de fórmulas, os professores deixarão de explorar as concepções espontâneas, que os alunos trazem antes de conhecer o conceito em questão, e nem estariam utilizando a ferramenta de aprendizagem adequada para atingir o objeto da aprendizagem.

Paralelamente, o conceito de semelhança de triângulo é importante nas resoluções de problemas de Geometria. Além disso, do ponto de vista matemático, esse conceito é muito importante por se constituírem pré-requisito para o estudo de vários conteúdos geométricos, assim como a grande riqueza de conceitos que ele próprio envolve (PEREIRA; PEREIRA, 2016). Em Pereira (2016) faz-se a seguinte abordagem sobre a importância do ensino:

A importância do ensino de semelhança de figuras planas pode ser notada tanto nos estudos supracitados quanto, quanto em documentos oficiais, que recomendam sua inserção deste conceito nos currículos escolares de matemática dos diferentes níveis do ensino, mas qual opinião de alunos em relação à aprendizagem deste tema? Qual a opinião de professores da educação básica em relação ao ensino de semelhança de figuras planas? O que dizem os estudos recentes a respeito das dificuldades no ensino de geometria, e quais são as novas abordagens metodológicas propostas por estes estudos? Quais efeitos o desenvolvimento de uma sequência didática pode provocar em alunos do $9^{\circ}$ ano do ensino fundamental, em relação à participação nas aulas de matemática e ao 
desempenho na resolução de questões envolvendo semelhança de figuras planas? E é a partir destas proposições que se precisa guiar a pesquisa.

Observa-se que é essencial para o ensino de semelhança de triângulo e áreas de figuras planas, que o professor procure alternativas metodológicas que melhorem a aprendizagem dos alunos para que os mesmos consigam estabelecer relações entre as equações e as projeções gráficas com ênfase nos conceitos de base, como por exemplo, o quadrado e seus lados, a altura do trapézio com suas bases, e a congruência entre as figuras planas (PAIS, 2002).

Por fim, a matemática é um instrumento que permite a percepção e a visualização do espaço. Pode desenvolver habilidades e auxiliar em outras áreas do conhecimento e têm muitas aplicações no mundo real. Explorações, representações e construções ajudam o estudante a investigar, descrever, perceber propriedades e, na elaboração de uma linguagem escrita correta e essencial, invade vários conceitos.

\subsection{Tecnologia na Educação: uma possibilidade eficiente com o GeoGebra}

Nos dias que correm, a ascensão dos meios eletrônicos multifuncionais portáteis tais como notebooks, celulares, smartphones, tabletes, entre outros recursos, facilitam o surgimento de programas, softwares e aplicativos educacionais. Essa nova realidade digital corrobora de modo direto com as interações de ensino e aprendizagem (GOODWIN; BOGUTCHI, 2016).

Para Mill (2018, p. 27) a relação entre ensino, aprendizagem, tecnologia são essenciais pela homogeneidade e a discussão de extrema notabilidade, conforme o fragmento:

Seja na Educação Presencial ou na Educação a Distância (EaD), pensar a relação entre o ensino e aprendizagem e as tecnologias é algo instigante e necessário, sobretudo porque são duas facetas indissociáveis - chega a ser uma relação (quase) simbiótica, sinérgica e biunívoca. Desta forma, refletir sobre a relação entre Educação e Tecnologia constitui uma discussão necessária e importante, na medida em que, desde suas origens, a Educação lançou mão de diferentes dispositivos tecnológicos como forma de melhoria do processo de ensino e aprendizagem. 
Para a Base Nacional Comum Curricular (BNCC), as tecnologias digitais e a computação são de extrema presentes nas nossas vidas e, consequentemente, para a educação básica:

\begin{abstract}
A contemporaneidade é fortemente marcada pelo desenvolvimento tecnológico. Tanto a computação quanto as tecnologias digitais de informação e comunicação (TDIC) estão cada vez mais presentes na vida de todos, não somente nos escritórios ou nas escolas, mas nos nossos bolsos, nas cozinhas, nos automóveis, nas roupas etc. Além disso, grande parte das informações produzidas pela humanidade está armazenada digitalmente. Isso denota o quanto o mundo produtivo e o cotidiano estão sendo movidos por tecnologias digitais, situação que tende a se acentuar fortemente no futuro.
\end{abstract}

A preocupação com os impactos dessas transformações na sociedade está expressa na BNCC e se explicita já nas competências gerais para a Educação Básica. Diferentes dimensões que caracterizam a computação e as tecnologias digitais são tematizadas, tanto no que diz respeito a conhecimentos e habilidades quanto atitudes e valores. (BRASIL, 2017, p. 473)

Com continuidade em Mill (2018, p. 35) evidencia a importância da ferramenta tecnológica mais adequada para o desenvolvimento do ensino e aprendizagem:

A tecnologia também levanta questões sobre a natureza dos próprios fins da educação - sobretudo porque a escolha de determinada tecnologia ( $\mathrm{e}$ não outra) para $\mathrm{o}$ ensino $\mathrm{e}$ aprendizagem representa um posicionamento do educador/gestor com significado peculiar. A própria forma como certa tecnologia é eleita diz muito nas entrelinhas dos propósitos da escolha. Certamente, os educadores, pesquisadores e gestores do campo da tecnologia educacional devem considerar essa natureza dos fins da educação.

Em seu trabalho Lieban e Muller (2012, p. 39-40), apresentam o software

GeoGebra como estratégia de ruptura de alguns vícios que permanecem nas 
metodologias atuais de ensino. No mesmo capítulo, discorrem do preparo que os educadores necessitam na aplicação da Geometria Dinâmica ${ }^{3}$ em sala de aula.

\begin{abstract}
Ao refletirmos sobre a utilização do GeoGebra como suporte à construção do conhecimento, podemos pensar sobre como se dá esta construção, ou ainda, como fazer uso desta ferramenta para quebrar a "tradição" de exercícios repetitivos e fórmulas.

Porém, para que o uso da Geometria Dinâmica possa contribuir na construção de uma aprendizagem significativa, é essencial que o professor sinta-se seguro para fazer uso da tecnologia, pois não é suficiente apenas disponibilizar ferramentas se a aplicação delas não for pensada e estudada previamente.
\end{abstract}

As primeiras percepções são que a ferramenta de educação, o conhecimento prévio sobre o software utilizado e o preparo de aulas podem ser diferenciais importantes para aulas de qualidade. Nesse quadro, apresenta-se um pouco da história do software GeoGebra ${ }^{4}$, criado em 2001 como tese de Markus Hohenwarter e seu reconhecimento como ferramenta educacional tem aumentado desde esse tempo. No momento atual, este software é usado em quase 200 países, traduzido para 55 idiomas.

O GeoGebra é um software de matemática dinâmica gratuito e multiplataforma para todos os níveis de ensino que reúne Geometria, Álgebra, Planilha de Cálculo, Gráficos, Probabilidade, Estatística e Cálculos Simbólicos em um único sistema fácil de se usar. Ele possui uma comunidade de milhões de usuários em praticamente todos os países e tornou um líder na área de softwares de matemática dinâmica, apoiando o ensino e a aprendizagem em Ciência, Tecnologia, Engenharia e Matemática. Algumas características interessantes a serem destacadas:

\footnotetext{
${ }^{3}$ A definição dada por Gravina et. al. (2011) foi que um software de Geometria Dinâmica é "uma mídia digital que disponibiliza régua e compasso virtuais, que são instrumentos clássicos com os quais são feitas as construções geométricas, só que agora em ambiente virtual”. (p.26).

${ }^{4}$ GEOGEBRA. Disponível em: https://www.geogebra.org. Nele se encontra, entre outros conteúdos atraentes, materiais e diversos aplicativos matemáticos para gráficos, geometria e 3D com acessos livres para os estudantes e professores interessados em saber mais sobre o programa.
} 
Geometria, Álgebra e Planilha de Cálculo estão interconectadas e são totalmente dinâmicas, logo, se pode modelar as mais várias circunstâncias e sugestões para resolução de problemas;

Interface fácil de se usar e, ainda assim, com muitos recursos poderosos;

Ferramentas de desenvolvimento para a criação de materiais didáticos como páginas web interativas;

Disponível em vários idiomas, facilitando milhões de usuários ao redor do mundo o acesso ao programa;

Software de Código Aberto disponível gratuitamente para usuários não comerciais.

\subsection{OBMEP}

A Olimpíada Brasileira de Matemática das Escolas Públicas - Obmep ${ }^{5}$ - é um projeto nacional dirigido às escolas públicas e privadas brasileiras, realizado pelo Instituto Nacional de Matemática Pura e Aplicada - Impa, com o apoio da Sociedade Brasileira de Matemática - SBM, e promovida com recursos do Ministério da Educação e do Ministério da Ciência, Tecnologia, Inovações e Comunicações - MCTIC.

Criada em 2005 para estimular o estudo da matemática e identificar talentos na área, a Obmep tem como objetivos principais:

$\checkmark$ Estimular e promover o estudo da Matemática;

$\checkmark$ Contribuir para a melhoria da qualidade da educação básica, possibilitando que um maior número de alunos brasileiros possa ter acesso a material didático de qualidade;

$\checkmark$ Identificar jovens talentos e incentivar seu ingresso em universidades, nas áreas científicas e tecnológicas;

$\checkmark$ Incentivar o aperfeiçoamento dos professores das escolas públicas, contribuindo para a sua valorização profissional;

${ }^{5}$ OLIMPÍADA BRASILEIRA DE MATEMÁTICA DAS ESCOLAS PÚBLICAS. Disponível: http://www.obmep.org.br/. Fonte de pesquisa sobre a Obmep é uma referência de pesquisa para os estudantes e professores interessados em saber mais sobre a olimpíada. Apresentação, Material Didático, Programas e Portais e Escolas Inscritas são alguns tipos de informações que são obtidas no site para ser mais sobre o que é esse programa. 
$\checkmark$ Contribuir para a integração das escolas brasileiras com as universidades públicas, os institutos de pesquisa e com as sociedades científicas;

$\checkmark$ Promover a inclusão social por meio da difusão do conhecimento.

O público-alvo da Obmep é composto de alunos do $6^{\circ}$ ano do Ensino Fundamental até último ano do Ensino Médio. Em 2018, mais de 18 milhões de alunos participaram da olimpíada.Ela promove vários programas com o objetivo de desenvolver o estudo da matemática e a inclusão social por meio do compartilhamento do conhecimento. Um exemplo foi a criação da Obmep nível $A$, que é uma Olímpiada voltada para os alunos do $4^{\circ}$ e $5^{\circ}$ ano do Ensino Fundamental das escolas públicas. Teve sua $1^{\text {a }}$ edição no ano de 2018 , e está prevista para ocorrer no $1^{\circ}$ semestre de 2019 , no mesmo formato do ano anterior ${ }^{6}$.

A seguir estão alguns programas desenvolvidos pela Obmep.

$\checkmark$ Obmep nível A;

$\checkmark$ Programa de Iniciação Científica JR. (PIC);

$\checkmark$ Portal da Matemática;

$\checkmark$ Banco de Questões e Provas Antigas;

$\checkmark$ Portal Clubes de Matemática;

$\checkmark$ POTI-Polos Olímpicos de Treinamento Intensivo;

$\checkmark$ PICME - Programa de Iniciação Científica e Mestrado;

$\checkmark$ Programa Obmep na Escola.

2. A QUESTÃo E A IMPORTÂNCIA DA VARIAÇÃo DAS SOLUÇÕES

\footnotetext{
6 Para mais informações sobre o programa Obmep nível A, acesse:www.obmep.org.br/informacoesNivelA.DO.
} 
Nesta seção, apresenta-se a questão que iniciou todo o desenvolvimento do trabalho com duas soluções: a primeira sobre uma relação matemática que afirma em todo triângulo retângulo a mediana relativa a hipotenusa é igual a metade da hipotenusa, enquanto, a segunda trata-se da solução oficial exibida pela Obmep.

\subsection{A Questão da Obmep}

A proposta pelo artigo consentiu na análise da questão da $13^{a}$ Olimpíada Brasileira de Matemática das Escolas Públicas (OBMEP) de 2017, da $1^{\text {a }}$ fase, nível 3 do ensino médio. Na prova foi a $13^{\text {a }}$ questão que se encontra abaixo:

Na figura, os ângulos $A \hat{B} C$ e $B \hat{C} D$ medem $120^{\circ}$, o ângulo $B \hat{A} D$ é reto, e os segmentos $B C$ e $C D$ medem $4 \mathrm{~cm}$ e $8 \mathrm{~cm}$, respectivamente. Qual é a área do quadrilátero $A B C D$ em $\mathrm{cm}^{2}$ ?

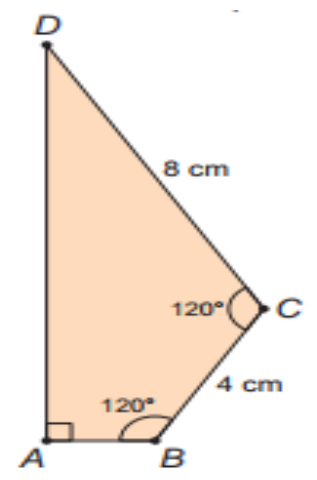

Desde já, credita-se o desenvolvimento das figuras para a solução pelo software matemático GeoGebra para facilitar o entendimento e interpretação.

\subsubsection{Variação de Soluções na Geometria}

Conforme mencionado anteriormente a primeira proposta de solução será: em todo triângulo retângulo a mediana relativa à hipotenusa é igual a metade da hipotenusa. Uma forma de deixar claro será um exemplo válido para encontrar essa propriedade. Para demonstrar esse fato considere o triângulo ABC retângulo em $\widehat{\boldsymbol{B}}$, de lados 3,4 e $5 \mathrm{~cm}$. 


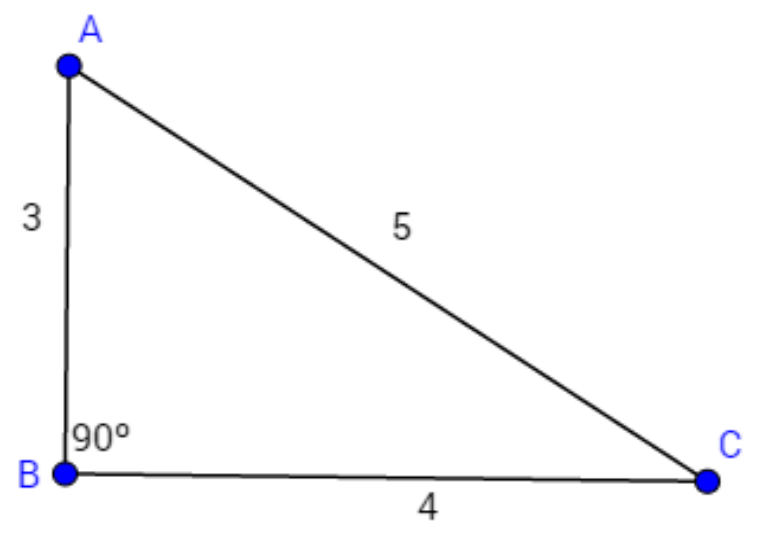

Agora a ideia é fazer a partir do triângulo original um retângulo de lados 3 $\mathrm{cm}$ e $4 \mathrm{~cm}$. Como mostra a figura a seguir.

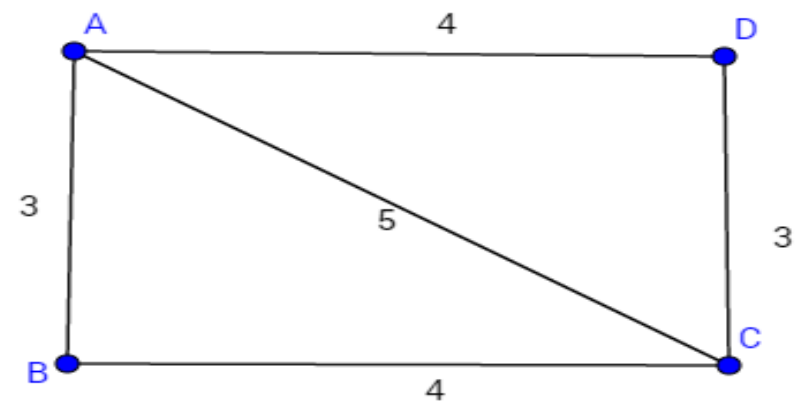

O Lado $\overline{\boldsymbol{A C}}$ é a hipotenusa e a diagonal do retângulo ABCD.Traçando outra diagonal que também mede $5 \mathrm{~cm}$, como mostra a figura abaixo, temos o retângulo $\mathbf{A B C D}$ com diagonais $\overline{\boldsymbol{A C}}, \overline{\boldsymbol{B D}}$ e se a diagonal mede $5 \mathrm{~cm}$, sua metade será $2,5 \mathrm{~cm}$.

Conclui-se que a mediana relativa a hipotenusa será $2,5 \mathrm{~cm}$. 


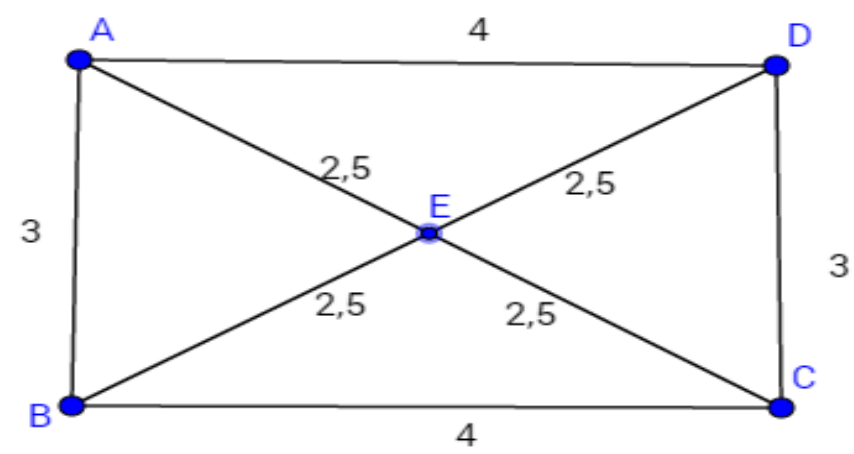

\section{PROBLEMA}

Na figura, os angulos $A \hat{B} C$ e $B \hat{C} D$ medem $120^{\circ}$, o angulo $B \hat{A D}$ é reto, e os segmentos $B C$ e $C D$ medem $4 \mathrm{~cm}$ e $8 \mathrm{~cm}$, respectivamente. Qual é a área do quadrilátero $A B C D$ em $\mathrm{cm}^{2}$ ?

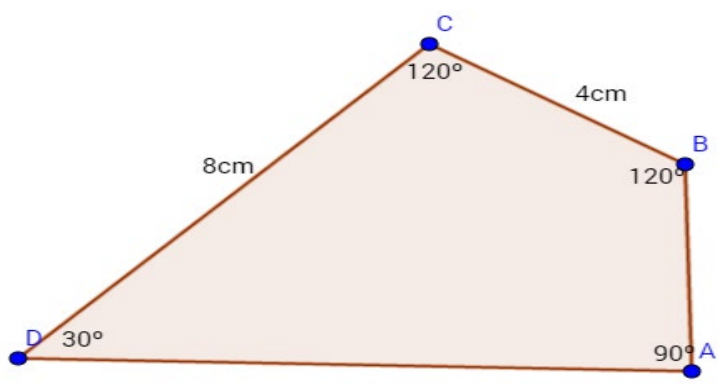

Solução:

Primeiramente, traçamos a altura de $\overline{\boldsymbol{C}}$ e, em seguida, a mediana $\overline{\boldsymbol{E H}}$.O lado $\overline{\boldsymbol{D C}}$ foi dividido em partes iguais. Como consequência temos que o triângulo HCE é equilátero, pois a mediana é metade da hipotenusa. Se a hipotenusa tem medida $8 \mathrm{~cm}$ e a mediana é metade, logo, a medida é $4 \mathrm{~cm}$.

Fazendo seno de $30^{\circ}$ no Triângulo DCE, temos:

$\frac{1}{2}=\frac{C E}{8}$ 
$2 C E=8$

$\overline{\boldsymbol{C E}}=4 \mathrm{~cm}$

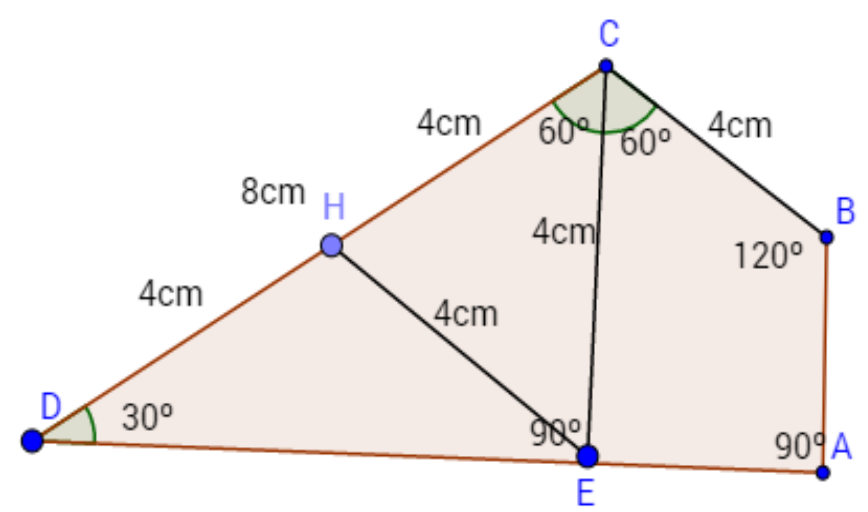

Agora traçamos o segmento $\mathrm{EB}$ (paralelo ao $\mathrm{HC}$ ) e temos que o $\Delta_{\mathrm{CEB}}$ também é equilátero, pois $\mathrm{HCEB}$ é um losango, logo, $\mathrm{EB}=4 \mathrm{~cm}$ e utilizando a relação do cosseno de $30^{\circ}$ no $\Delta_{\mathrm{DCE}}$, temos:

$$
\begin{aligned}
& \frac{\sqrt{3}}{2}=\frac{D E}{8} \\
& 2 D E=8 \sqrt{3} \\
& \overline{\boldsymbol{D E}}=4 \sqrt{3} \mathrm{~cm}
\end{aligned}
$$

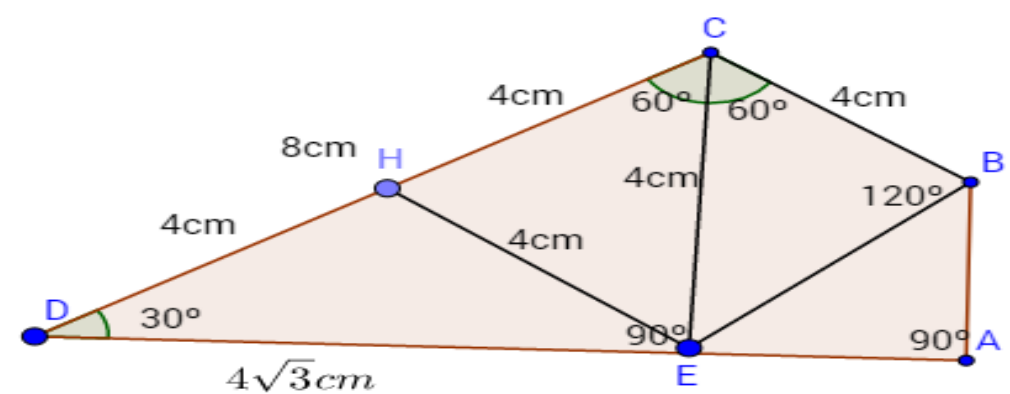


O triângulo CHE é equilátero e também o triângulo CBA(L.A.L) e seus ângulo são todos iguais a $60^{\circ}$.O triângulo DCE e o triângulo EBA são semelhantes, pois tem ângulos iguais e lados proporcionais.

$\frac{D C}{D E}=\frac{B E}{E A}$

$\frac{8}{4 \sqrt{3}}=\frac{4}{E A}$

4.4. $\sqrt{3}=8 \cdot E A$

$\overline{\boldsymbol{E A}}=2 \sqrt{3} \mathrm{~cm}$

Aplicando novamente a semelhança tem-se:

$$
\frac{D C}{C E}=\frac{B E}{B A}
$$

$\frac{8}{4}=\frac{4}{B A}$

$\overline{\boldsymbol{B A}}=2 \mathrm{~cm}$

Somando agora as áreas dos $\Delta_{\mathrm{DCE}}, \Delta_{\mathrm{CBE}} \mathrm{e} \Delta_{\mathrm{EBA}}$ para a formação da área total do quadrilátero, temos:

$$
\begin{aligned}
& \hat{\mathrm{A}} R E A_{T O T A L}=\hat{\mathrm{A}} R E A_{\triangle D C E}+\hat{\mathrm{A}} R E A_{\triangle C B E}+\hat{\mathrm{A}} R E A_{\triangle E B A} \\
& \text { ÁREA TOTAL }=\frac{4 \sqrt{3} \cdot 4}{2}+\frac{4^{2} \sqrt{3}}{4}+2 \cdot \frac{2 \cdot \sqrt{3}}{2} \\
& \text { ÁREA } A_{\text {TOTAL }}=8 \sqrt{3}+4 \sqrt{3}+2 \sqrt{3} \\
& \text { ÁREA } A_{\text {TOTAL }}=14 \sqrt{3} \mathrm{~cm}^{2}
\end{aligned}
$$

\subsubsection{Solução Oficial Obmep}

A solução 13 está no site oficial da Obmep, no qual observa-se a solução e o vídeo sobre a prova do ano de 2017.A ideia desenvolvida foi por 
prolongamentos até a formação de um triângulo equilátero e, a partir disso, remover as áreas acrescentadas para a formação do triângulo equilátero e, assim, se obter a área do quadrilátero pedido na questão. Em Sessa $(2009$, p. 17) pode-se fazer uma reflexão sobre a adição de áreas:

Essa consideração geométrica da operação "produto" atravessa a matemática desde a Antiquidade até Descartes (1596-1650) e nos leva a considerar unicamente adições que envolvam objetos da mesma "dimensão": não posso somar [a área de] um quadrado a [o comprimento de] um segmento, mas sim [área de] um quadrado a [área de] um retângulo. É o que se conhece como princípio da homogeneidade, que regerá o trabalho algébrico de suas origens até o grande salto que se produz com a geometria cartesiana.

\section{PROBLEMA}

Na figura, os ângulos $A B C$ e $B C D$ medem $120^{\circ}$, o angulo $B \hat{A D}$ é reto, e os segmentos $B C$ e $C D$ medem $4 \mathrm{~cm}$ e $8 \mathrm{~cm}$, respectivamente. Qual é a área do quadrilátero $A B C D$ em $\mathrm{cm}^{2}$ ?

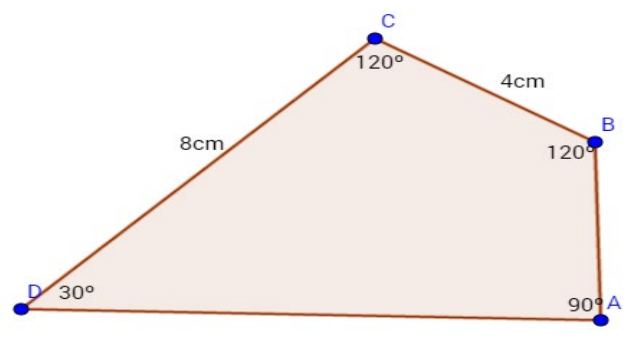

Solução:

Inicialmente, prolongam-se dois lados do quadrilátero e obtemos um triângulo equilátero de lado $4 \mathrm{~cm}$ e um triângulo retângulo grande DAE que é metade de um triângulo equilátero de lado $12 \mathrm{~cm}$, como indicado na figura. Pelo Teorema de Pitágoras, a altura de um triângulo equilátero de lado 1 é $\mathrm{h}=\frac{\sqrt{3}}{2} . l$, então:

$h=\frac{\sqrt{3} \cdot l}{2}$ 


$$
\begin{aligned}
& h=\frac{\sqrt{3} .12}{2} \\
& h=6 \sqrt{3} \mathrm{~cm}
\end{aligned}
$$

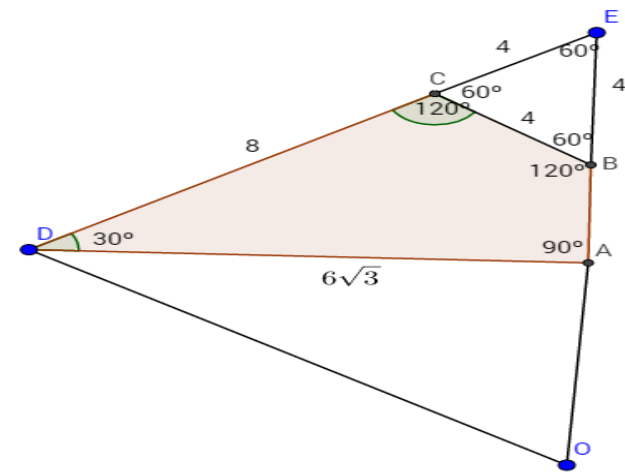

Logo, área do quadrilátero $\mathbf{A B C D}$ é igual a área do triângulo retângulo DAE menos a área do triângulo equilátero CBE, então:

$$
\begin{aligned}
& \text { ÁRE } A_{Q U A D R I L A ́ T E R O ~ A B C D}=\hat{A} R E A_{\triangle D A E}-\hat{A} R E A_{\triangle C B E} \\
& \text { ÁREA } A_{T O T A L}=\frac{6 \sqrt{3} .6}{2}-\frac{4^{2} \sqrt{3}}{4} \\
& \text { ÁREA } A_{\text {TOTAL }}=18 \sqrt{3}-4 \sqrt{3} \\
& \text { ÁREA } A_{\text {TOTAL }}=14 \sqrt{3} \mathrm{~cm}^{2} \text {. }
\end{aligned}
$$

\section{CONSIDERAÇÕES FINAIS}

O que é uma questão fácil ou difícil? Estes conceitos são relativos e podem mudar na concepção dos estudantes, sendo assim não se pode mensurar esses dois conceitos na vida estudantil. Na matemática tanto na álgebra quanto na geometria é possível encontrar variadas soluções para um mesmo problema e esse, sem dúvida, é um dos motivos que fazem com que a matemática seja considerada por muitos como a "Rainha das Ciências", em que um único problema podem levar a caminhos (soluções) diferentes, porém, com a mesma resposta o que torna essa ciência tão desafiadora e apaixonante ao mesmo tempo. 
Por isso, a variação de soluções pode ser muito importante e quando oportuno o professor pode e deve usufruir desse "artifício" com intuito de contemplar um assunto ou mesmo um problema matemático ao maior número de alunos possível. A geração atual gosta de desafios e usufruem da tecnologia para solucionar esses problemas.

Nessa linha, após as soluções apresentadas no artigo, os autores tiveram a sensação de trabalho incompleto, devido a questão da Obmep (13 ${ }^{\circ}$ questão da prova de 2017, Nível 3, da $1^{\text {a }}$ fase) disponibilizar uma diversificação de soluções tornando-se um verdadeiro quebra-cabeça. Com isso, um livro foi escrito abordando 13 soluções encontradas - GeoGebra Soluções na Geometria - que trata-se de temas trabalhados neste artigo e outras curiosidades.

Vale salientar que o uso da tecnologia não diminui o papel do professor, ao contrário, estas ferramentas auxiliam os professores em suas vidas escolares e permite diminuir em alguns casos a distância entre professores e alunos. Tenta-se deixar evidente que o professor é um dos pilares da educação e fator inigualável para a melhoria da educação no nosso país.

O uso do GeoGebra foi predominante para a demonstração de como a tecnologia pode se impor no ensino. O planejamento de utilização desta ferramenta possibilita a perfeita harmonia entre professor, conteúdo e aluno. O uso da tecnologia está crescendo, cada vez mais, no mundo, e claro, no Brasil e nos anos que se sucederão ela estará ainda mais presente não somente nas salas de aula, mas também em nossas vidas.

\section{REFERÊNCIAS}

BRASIL. MEC. Base Nacional Comum Curricular. Brasília, 2017.

CHIUMMO, A. Conceito de áreas de figuras planas: capacitação de professores do ensino fundamental.181f. Dissertação (Mestrado em Educação matemática) Pontifícia Universidade Católica de São Paulo, 1998. 
DEVLIN, K.O gene da Matemática. Editora Record. Rio de Janeiro.2008.

DUVAL, R. Approche cognitive des problemes de geometrie em termes de congruence.Revista Annales de Didactique et de sciences cognitives, Paris, v. 01, p. 57-74, 1988.

DZIADZIO, J. R.; VAN KAN, M. T. Percepções e relações da geometria espacial com o cotidiano dos alunos. In: Os desafios da escola pública paranaense na perspectiva do professor PDE. vol. 1. Paraná/PR: Governo do estado do Paraná, 2016.

FAINGUELERNT, E. K.; NUNES, K. R. A. Matemática: práticas pedagógicas para o ensino médio. Porto Alegre: Penso. 2012. 162 p.

FERREIRA, A. B. H. Miniaurélio: o dicionário da língua portuguesa. 8. ed. Curitiba: Positivo, 2010. 895 p.

GOODWIN, F. C.; BOGUTCHI, T. F. Uso do GeoGebra por meio do tablet no estudo das funções. In: ENCONTRO NACIONAL DE EDUCAÇÃO

MATEMÁTICA (ENEM), 12, 2016. São Paulo. Anais... São Paulo: Enem, 2016.

GRAVINA, M.A., et. al. Geometria Dinâmica na Escola. In: GRAVINA, M.A. et. al. (Orgs). Matemática, Mídias Digitais e Didática - tripé para a formação de professores de Matemática. Porto Alegre: Editora da UFRGS, 2011, p. 26-45.

HOLANDA FILHO, I. O.; CRUZ, M. P. M. GeoGebra: Soluções na Geometria. Curitiba: Appris, 2019. 113 p.

LIEBAN, D. E.; MULLER, T. J. Construção de utilitários com o software GeoGebra: uma proposta de divulgação da geometria dinâmica entre professores e alunos. In: CONFERÊNCIA LATINO AMERICANA DE GEOGEBRA

(GEOGEBRA-LA-2011), 1, 2012. São Paulo. Anais... São Paulo: (GeoGebra-la2011), 2012.

LIMA, J. A.; SAMPAIO, C. G.; BARROSO, M. C. S. Contribuições de uma ueps com experimentação para aprendizagem de funções orgânicas: percepção dos alunos. In: VASCONCELOS, F. H. L.; SAMPAIO, C. G.; VASCONCELOS, A. K. P.; BARROSO, M. C. S. (Org.). Ensino de Ciências e Matemática: Experiências da formação continuada de professores na Pós-graduação do IFCE. Campinas/SP: Pontes Editores, 2018. p. 14-31.

LIMA, M. V. M.; ALVES, F. R. V.; SALES, G. L. Uma engenharia didática para o ensino de integrais dependentes de parâmetros: análises preliminares e análise a priori. In: VASCONCELOS, F. H. L.; SAMPAIO, C. G.; VASCONCELOS, A. K. P.; BARROSO, M. C. S. (Org.). Ensino de Ciências e Matemática: Experiências da formação continuada de professores na Pós-graduação do IFCE. Campinas/SP: Pontes Editores, 2018. p. 162-177. 
LIMA, T. S.; PEREIRA, A. C. C.; Percepções dos participantes do curso de extensão sobre o vídeo como recurso didático para o ensino da matemática. In: VASCONCELOS, F. H. L.; SAMPAIO, C. G.; VASCONCELOS, A. K. P.; BARROSO, M. C. S. (Org.). Ensino de Ciências e Matemática: Experiências da formação continuada de professores na Pós-graduação do IFCE. Campinas/SP: Pontes Editores, 2018. p. 178-195.

LORENZATO, S. Por que não ensinar geometria?.Educação Matemática em Revista, SBEM, Blumenau, n. 04, p. 3-13, jan./mar.,1995.

MILL, D. Reflexões sobre a relação entre educação e tecnologias: algumas aproximações. In: CAVALCANTE, M. J. M.; HOLANDA, P. H. C.; TORRES, A. L. M. M. (Org.). Tecnologias da educação: passado, presente, futuro. Fortaleza/CE: Edições UFC, 2018. p. 27-47

PAVANELLO, R. M. (1989). O abandono do ensino da geometria: uma visão histórica. 1989. 195 f. Dissertação (Mestrado em Educação) - Faculdade de Educação, Universidade Estadual de Campinas, Campinas.

PAIS, L.C. Didática da Matemática - Uma análise da influência francesa.2.ed. Belo Horizonte: Autêntica, 2002. (Coleção Tendências da Educação Matemática).

PEREIRA, M.F.F. Uma sequência didática para o ensino de semelhança de figuras planas.In:Encontro Brasileiro de Estudantes de Pós-Graduação em Educação Matemática (EBRAPEM), 20, 2016, Curitiba. Anais... Curitiba: Ebrapem, 2016.

PEREIRA, S.R.F; PEREIRA, M.F.F. O ensino de semelhança de triângulos na opinião de alunos.In:Encontro Nacional de Educação Matemática. Educação Matemática na Contemporaneidade: desafios e possibilidades. São Paulo, 13 a 16 de julho de 2016.

PEREZ, G. Pressupostos e reflexões teóricos e metodológicos da pesquisa participante no ensino de geometria para as camadas populares. $1991.298 \mathrm{f}$. Tese (Doutorado em Educação) - Faculdade de Educação, Universidade Estadual de Campinas, Campinas, 1991.

PIRES, C. M. C. Currículos de Matemática: da organização linear à ideia de rede. São Paulo: FTD, 2000. 223 p.

RODRIGUES, J. M.; ALVES, R. F. Fanzine no ensino de biologia: (re)criando uma ponte entre a ciência e a arte. In: VASCONCELOS, F. H. L.; SAMPAIO, C. G.; VASCONCELOS, A. K. P.; BARROSO, M. C. S. (Org.). Ensino de Ciências e Matemática: Experiências da formação continuada de professores na Pósgraduação do IFCE. Campinas/SP: Pontes Editores, 2018. p. 78-95.

ROGENSKI, M. L. C.; PEDROSO, S. M. D. O ensino da geometria na educação básica: realidade e possibilidades. 2007. Disponível em:

http://www.diaadiaeducacao.pr.gov.br/portals/pde/arquivos/44-4.pdf acessado no dia 05/05/2019 às 11:30 horas. 
SANTOS, A. A.; ALVES, F. R.; VASCONCELOS, F. H. L. A engenharia didática em completude com a teoria das situações didáticas como percurso metodológico. In: VASCONCELOS, F. H. L.; SAMPAIO, C. G.; VASCONCELOS, A. K. P.; BARROSO, M. C. S. (Org.). Ensino de Ciências e Matemática: Experiências da formação continuada de professores na Pósgraduação do IFCE. Campinas/SP: Pontes Editores, 2018. p. 210-227.

SANTOS, W.S.; JUCÁ, R.S. O ensino das áreas das figuras planas com a utilização do software kig. Revista WEB-MAT, Belém, v. 01, n. 01, jan./jun., 2014.

SARAIVA, F. A.; VASCONCELOS, A. K. P.; BARROSO, M. C. S. Teoria da aprendizagem significativa: aspectos bibliográficos aplicados ao ensino de química. In: VASCONCELOS, F. H. L.; SAMPAIO, C. G.; VASCONCELOS, A. K. P.; BARROSO, M. C. S. (Org.). Ensino de Ciências e Matemática: Experiências da formação continuada de professores na Pós-graduação do IFCE. Campinas/SP: Pontes Editores, 2018. p. 32-49.

SILVA, F. R. O.; ROMEU, M. C.; GONÇALVES, N. S. Aprendizagem de conceitos da cinemática utilizando a tecnologia RFID e a plataforma arduino. In: VASCONCELOS, F. H. L.; SAMPAIO, C. G.; VASCONCELOS, A. K. P.; BARROSO, M. C. S. (Org.). Ensino de Ciências e Matemática: Experiências da formação continuada de professores na Pós-graduação do IFCE. Campinas/SP: Pontes Editores, 2018. p. 148-159.

VASCONCELOS, P. H. M.; ARAÚJO, A. F. S. Contribuições da experimentação e da contextualização para o ensino da cinética química. In: VASCONCELOS, F. H. L.;

SAMPAIO, C. G.; VASCONCELOS, A. K. P.; BARROSO, M. C. S. (Org.). Ensino de Ciências e Matemática: Experiências da formação continuada de professores na Pós-graduação do IFCE. Campinas/SP: Pontes Editores, 2018. p. 50-64. 